\title{
Syntaxe en interaction et variation diaphasique : l'exemple des dislocations dans ESLO2
}

\author{
Luka Raickovic ${ }^{1}$, Marie Skrovec ${ }^{1}$ \\ ${ }^{1}$ Université d'Orléans - LLL UMR 7270
}

\begin{abstract}
Résumé. La dislocation en tant que dispositif syntaxique se caractérise par la présence d'un syntagme à la périphérie d'une structure phrastique, au sein de laquelle celui-ci conserve généralement une trace sous une forme pronominale. Cette construction, qui connaît de nombreuses variantes, a été décrite aussi bien à l'écrit qu'à l'oral, et tout en étant globalement peu fréquente, est souvent considérée comme caractéristique de l'oral. L'oral ne formant pas un ensemble homogène, la présente étude se propose d'examiner ce phénomène dans un corpus oral échantillonné issu des Enquêtes Sociolinguistiques à Orléans (ESLO). A partir d'un relevé systématique et d'une annotation manuelle de leurs propriétés syntaxiques et pragmatiques, nous cherchons à estimer la distribution des dislocations au sein d'une sélection de contextes interactionnels différents, illustrant les pratiques orales des plus formelles aux plus spontanées, afin de mettre en évidence un effet du contexte interactionnel sur le mode de structuration des énoncés à l'oral. Par ailleurs, notre travail offre des pistes pour l'analyse de l'emploi du format disloqué dans la réalisation des tâches interactionnelles spécifiques comme la préclôture topicale ou les séquences narratives.
\end{abstract}

\begin{abstract}
Syntax in interaction and diaphasic variation: the case of dislocations in ESLO2. Dislocations as a syntactic phenomenon is characterized by the presence of a syntagm at the periphery of a phrasal structure, within which it is usually represented by a pronoun. This construction, which knows many variants, has been described in both the written and the spoken language, and while generally infrequent, is often considered characteristic of the spoken language. As the spoken language does not form a homogeneous whole, the present study aims to examine this phenomenon in a sampled spoken corpus built from the Enquêtes Sociolinguistiques à Orléans (ESLO). From a systematic survey and manual annotation of their syntactic and pragmatic properties, we aim to estimate the distribution of dislocations within a selection of different interactional contexts, illustrating oral practices from the most formal to the most spontaneous, in order to highlight an effect of the interactional context on the way spoken utterances are structured. Moreover, our work offers leads for the analysis of the use of the dislocations in the realization
\end{abstract}


of specific interactional tasks such as topic preclosing or narration in dialogs.

\section{Introduction}

L’importance des fonctions syntaxiques périphériques est souvent citée comme une caractéristique structurelle majeure du français oral. Au sein de cette zone périphérique, différents objets retiennent l'attention des chercheurs, comme la dislocation, souvent considérée comme typique de l'oral informel, résultant d'un ordre de mot dit « expressif» (Bally 1909 : 313) s'écartant de l'ordre dit « logique » (ibid. : 313). Si la littérature permet de dresser un inventaire très fourni des différents types de dislocations (voir notamment Blasco-Dulbecco 1999), et d'accéder à des analyses qualitatives fines qui mettent en évidence différents cas de figure (dislocation à gauche ou à droite, dépendance ou indépendance syntaxique de l'élément disloqué, lien référentiel plus ou moins fort avec le syntagme disloqué, structuration topicale et informationnelle, lien avec la dynamique interactionnelle, etc.), il manque sur cet objet, nous semble-t-il, une étude qui soit à la fois quantitative et fondée sur des données variées et situées au sein d'un corpus structuré. Le présent travail propose, à partir d'un relevé et d'une annotation systématiques des cas de dislocations dans un corpus oral, d'examiner leur distribution au sein d'une sélection de contextes interactionnels différents, issus du corpus ESLO (Enquêtes Sociolinguistiques à Orléans). Pour mieux cerner notre objet d'étude, nous passerons en revue différents travaux sur les dislocations avant de présenter le corpus d'étude et la méthodologie. La base de données ESLO fournit en effet un réservoir permettant de sélectionner, dans une grande quantité de données, des enregistrements illustrant les pratiques orales des plus formelles aux plus spontanées. L'annotation systématique des traits caractérisant les dislocations (nature et catégorie syntaxique des éléments disloqués, organisation topicale et structure de l'information) permet, outre de préciser la fréquence des structures et des types d'emplois, de mettre en évidence l'effet du contexte interactionnel sur le mode de structuration des énoncés à l'oral. Nous verrons en effet que la distribution des différents types de dislocations reflète le continuum variationnel entre proximité et distance communicative.

\section{Les dislocations en français parlé contemporain : vers une définition de l'objet}

La dislocation en français en tant que phénomène linguistique a été étudiée par de nombreux chercheurs appartenant à différentes écoles de pensée. Récemment, Avanzi (2009 : 79) ou encore Berrendonner (2015) ont passé en revue ces différents travaux. Ils rappellent que ces auteurs, adoptant différents angles d'attaque afin de mieux cerner ce phénomène, se sont penchés tour à tour sur son évolution diachronique (Marchello-Nizia 1998, Prévost 2003), ses aspects syntaxique (par ex. Blasco-Dulbecco 1999, Berrendonner \& Béguelin 1997), interactionnel (par ex. Pekarek-Doehler 2001), prosodique (par ex. Rossi 1999), psycholinguistique et acquisitionnel (De Cat 2007), ainsi que son lien avec la structure informationnelle (par ex. Lambrecht 1981), etc.

L'intérêt pour ce phénomène s'explique entre autres par les problèmes définitoires qu'il pose et l'enjeu de sa délimitation. La définition première est généralement donnée à partir d'une analyse syntaxique basée sur une conception canonique de la structure propositionnelle. Ainsi, selon le point de vue générativiste de Milner (1989 : 403), chaque élément de la phrase a sa place canonique au sein de la proposition. La présence d'un élément en dehors de cette place, soit en périphérie gauche, soit à droite de la phrase, est 
donc considérée comme non canonique, et traditionnellement appelée dislocation, terminologie largement reprise en linguistique, même en dehors du paradigme générativiste (Berrendonner $2015: 1$ ).

(1) cette histoire on la connaît (Blasco-Dulbecco 1999 : 39)

La dislocation stricto sensu se caractérise donc par le mouvement d'un syntagme en dehors de la structure canonique, au sein de laquelle celui-ci conserve une trace sous une forme pronominale. Le syntagme nominal disloqué est ainsi repris par un pronom coréférentiel anaphorique (dislocation à gauche) ou cataphorique (dislocation à droite). La dislocation prototypique porte ainsi sur les arguments du verbe (sujet et compléments régis), et ne concerne pas les modifieurs de phrase. Mais l'exemple (1), illustration. de la dislocation stricto sensu, constitue finalement une variante d'un phénomène plus vaste regroupant un ensemble de constructions aux caractéristiques proches. La définition du phénomène de la dislocation dans son intégralité pose en effet plusieurs problèmes, dans la mesure où il s'agit d'un type de construction regroupant des structures hétérogènes. Ainsi, tous les cas décrits comme des dislocations dans la littérature ne comportent pas nécessairement de reprise de l'élément disloqué. C'est sur le plan syntaxique surtout que se manifeste cette hétérogénéité. On considérera en effet comme des dislocations aussi bien des constructions dans lesquelles une place de rection est marquée deux fois par des éléments différents (Blasco-Dulbecco 1999 : 209) comme en (2), ou des énoncés dans lesquels les différents adjoints au pronom clitique se situent sur un continuum de référentialité de différents degrés, comme les yaourts et ça en (3), ainsi que les adjoints à l'ensemble de la construction verbale sans reprise pronominale (4), (idem 1999: 110, $111){ }^{1}$

(2) les travailleurs sociaux de plus en plus ils sont priés de devenir des agents du pouvoir ESLO2 INTPERS 1245

(3) les yaourts ça ressemble aux compotes

ESLO2_REPAS_1257

(4) c'était son grand-père les yeux bleus

ESLO2_REPAS_1256

En (4), l'élément disloqué à droite n'est pas repris par un pronom dans le noyau phrastique. Par conséquent, il s'agit d'une construction bien décrite dans la littérature sous le nom de nominativus pendens (Havers 1925), ou plus récemment de hanging topic (Grosu 1975, Cinque 1977), ou encore couplages (Deulofeu 1977) ou Chinese-style topics (Chafe 1976) (cf. Horlacher 2015).

Du point de vue pragmatique, la dislocation est considérée de manière unanime (Berrendonner 2015), comme un procédé de topicalisation c'est-à-dire comme un format réalisant une opération sur la structure informationnelle d'un énoncé en promouvant un référent au statut de topique, (encore appelé thème). Pour définir le topique, on peut retenir la notion d"aboutness" proposée par Lambrecht (1996 : 117-131), c'est-à-dire le propos en cours, complétée par la définition suivante de Berrendonner :

Bien que la notion de thème/topique soit éminemment floue, on s'accorde en général sur l'idée qu'un constituant thématique (i) est " hors focus », non porteur d'information inédite, et (ii) renvoie à un référent " donné », c'est-

\footnotetext{
1 Il est à noter que Berrendonner $(2015,1.2 .5)$ propose de distinguer, à partir de critères essentiellement prosodiques, la dislocation à gauche prototypique du phénomène de double marquage $\mathrm{du}$ sujet comme variante populaire de la structure canonique (les gens ils viennent pas). Pour ce dernier, le double marquage n'est pas une dislocation mais une variante vernaculaire témoignant d'une spécificité des clitiques en français oral, qui tendraient à se comporter davantage comme des affixes. Tout en convenant de l'intérêt de la distinction, notamment pour l'analyse morphosyntaxique, nous avons préféré considérer tous les cas de double marquage comme des dislocations, dans une conception du phénomène inspirée des travaux de Blasco-Dulbecco.
} 
à-dire identifiable pour l'allocutaire, parce que compris dans l'état courant du savoir partagé (ou présumé tel). En attribuant ce statut aux constituants disloqués, on rend compte de leurs principales propriétés sémantiques : ils ne peuvent être ni des quantifieurs, ni des mots interrogatifs (ceux-ci étant toujours focaux) ; ils sont hors du champ des modalités qui portent sur le noyau (négation, opérateurs restrictifs); ils ne peuvent apporter réponse à une question partielle (faute de pouvoir être interprétés comme focaux); et ils servent souvent à redésigner un référent qui vient d'être introduit dans le contexte antérieur, pour en faire le support d'un nouveau propos. (nous soulignons).

A contrario le focus, qui s'articule au topique, est l'information présentée comme nouvelle, celle sur laquelle on fait porter l'attention. Il est à noter que dans certains cas, certes plus rares, la dislocation n'isole pas le topique, mais le focus, comme dans l'exemple (5) où le propos nouveau, et donc en position de focus, se trouve dans l'élément disloqué (le précoce aussi), tandis que le topique correspond au segment il est sympa :

(5) FG719:

ils sont sympas ton frère ta soeur et euh enfin Alexandre et Clara et tout

le précoce aussi il est sympa

elle l'appelle pas Nico elle l'appelle le précoce [rire en fond]

ESLO2_REPAS_1265

Par ailleurs, si la dislocation englobe des structures non homogènes d'un point de vue syntaxique, il ne s'agit pas pour autant d'une catégorie fourre-tout, et il convient de délimiter le phénomène afin d'éviter la confusion avec des "structures limitrophes ", comme le focus movement, les termes d'adresse ou encore les compléments introduits par comme. Dans le premier cas, on observe une modification de la structure SVO à des fins d'expressivité, qui, associée à une prosodie spécifique (descendante sur le premier segment, basse plate sur le second, Berrendonner 2015), permet une focalisation sur le complément antéposé, pour reprendre le terme utilisé par Blanche-Benveniste (1996 : 113). Elle en donne les exemples suivants :

(6) 17 ans il a

(7) 10 centimes vous n'auriez pas

Dans le cas des termes d'adresse, c'est l'ancrage déictique qui distingue cette structure de la dislocation, l'opération effectuée par cette dernière relevant de la référence anaphorique, et non déictique. Ainsi, l'exemple suivant, qui comporte un nom propre en position extraphrastique et un pronom clitique coréférent intraphrastique, appartient à la catégorie des termes d'adresse, phénomène très bien décrit dans la littérature (Lagorgette 1998, Lehmann 2010). En effet, malgré l'existence d'un lien coréférentiel exact entre le nom propre et le clitique, nous avons choisi de nous rallier à un certain consensus dans le champ $^{2}$ et de ne pas prendre en compte ce type de structure, en raison de sa valeur déictique correspondant davantage à des fonctions conative et phatique que référentielle.

(8) WZ384: est-ce qu'y a un kiwi pour moi ? s'il te plaît Victor tu fais quoi là ? remets-toi à table j'ai pas mangé

ESLO2_REPAS_1254

Il en va de même pour les dislocations en «comme » que nous avons décidé d'écarter car le mot «comme» à notre sens ne peut pas être considéré comme déterminant, équivalent à un démonstratif par exemple (Nølke 1998 : 391) : il s'agit plutôt d'un élément de type prépositionnel permettant d'introduire la catégorie à laquelle le référent appartient (Lambrecht 1996b :193, cf. également Lambrecht 2003).

(9) DS952SOE: non mais elle elle est tarée comme fille quand même

\footnotetext{
${ }^{2}$ En effet, comme le rappelle Horlacher (2015: 55) il n'est pas habituel de traiter ce genre de construction en tant que dislocation.
} 


\section{ESLO2_REPAS_1256}

Plus exactement, dans l'exemple (9), bien qu'on puisse imaginer au même endroit la réalisation «elle est tarée cette fille », les deux énoncés ne sont pas identiques du point de vue sémantique.

Notre étude prend donc en compte les résultats des travaux précédemment évoqués en se focalisant sur l'aspect interactionnel ainsi que sur la variation diaphasique, et en se fondant sur la conception de Blasco-Dulbecco (1999) qui, grâce à son « échelle de relations référentielles ", décrit l'existence d'un continuum entre le double marquage qui instaure une dépendance syntagmatique maximale (10), et l'absence de relation syntagmatique, communément appelé le hanging topic (11 et 12).

(10) une chose pareille ! Voyons ! sérieusement, y songez-vous (Bally 1921 : 312)

que l'auteur oppose à une phrase dite "logique" Vous ne pouvez pas songer à une chose pareille.

(11) ce film ils auraient dû mettre plus de sang (Blasco-Dulbecco 1999 : 139)

(12) c'était même à la maison les deux langues (Horlacher $2015: 46$ )

Nous accorderons également une attention particulière aux dislocations pronominales du type «moi je » (Blasco-Dulbecco $2006: 29,30$ ), fréquente dans notre corpus et aux doubles dislocations (Horlacher 2015), comme dans l'exemple suivant où le même constituant est disloqué à la fois à gauche et à droite :

(13) la panure non ça se mange pas comme ça la panure

ESLO_REPAS_1257

\section{Corpus d'étude et méthodologie}

Le corpus d'étude est une sélection issue des Enquêtes Sociolinguistiques à Orléans, corpus conçu comme un réservoir de données orales situées, illustrant les pratiques du français contemporain hexagonal, en prenant pour contexte l'agglomération orléanaise. Collecté en deux campagnes menées à 40 ans d'intervalle (ESLO1 entre 1968 et 1974, ESLO2 depuis 2008), ce corpus présente la particularité de contenir, outre un grand nombre d'entretiens sociolinguistiques effectués auprès d'un panel de locuteurs sociologiquement diversifié, des échantillons prélevés dans une grande variété de contextes communicatifs. La composition actuelle du corpus (cf. schéma ci-dessous), dont une partie est en accès libre ${ }^{3}$, permet d'étudier de la parole produite dans des conditions communicatives variables illustrant une partie du continuum variationnel de l'oral, et donc d'interroger la variation diaphasique.

\footnotetext{
${ }^{3}$ Selon les estimations de Dugua (2019), le volume des données disponibles en ligne est de 5 millions de mots (http://eslo.huma-num.fr). Environ 2,5 millions de mots ne sont pas en libre accès.
} 


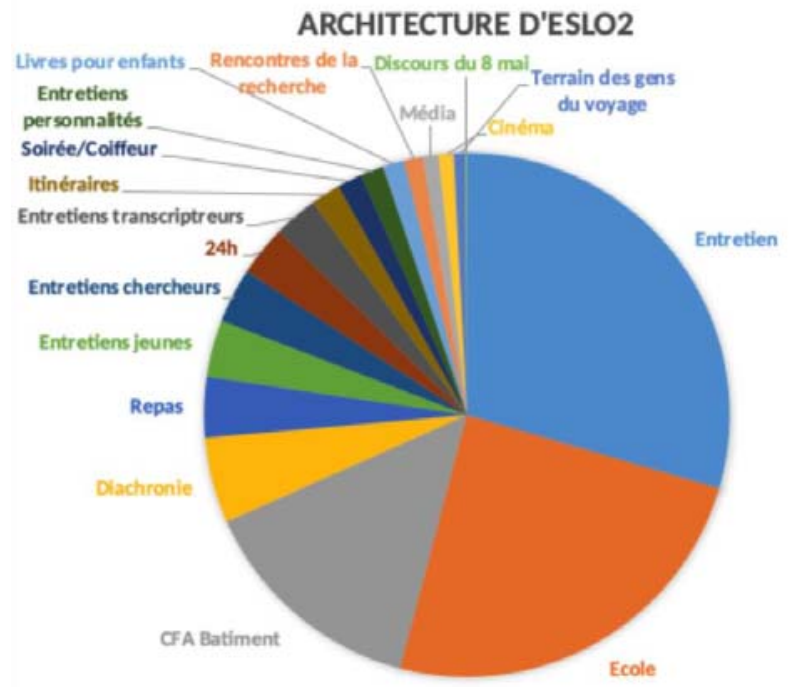

Fig. 1 : Composition actuelle du corpus ESLO2 par module

Le corpus d'étude, d'un volume total de 103557 mots, a été sélectionné de manière à illustrer différents degrés du continuum entre proximité et distance communicative ainsi que défini par Koch \& Oesterreicher (2001) à la suite de Söll (1974/1985) (cf. également Baude \& Guerin 2015). Les situations retenues, au nombre de 5, se caractérisent par un degré variable de familiarité des locuteurs, de formalité du cadre communicatif, d'interactivité et de planification thématique : le repas entre proches, la demande d'itinéraire dans la rue, le micro-trottoir à la sortie du cinéma, l'entretien avec des personnalités de la ville d'Orléans et la conférence universitaire nous ont semblé pouvoir être échelonnés au long de ce continuum. La taille des sous-corpus étant variable, chacun d'eux a fait l'objet d'une pondération afin d'augmenter leur comparabilité en prenant pour valeur de base le plus grand sous-corpus des repas. Cela nous a permis d'attribuer un poids relatif à chaque sous-corpus nécessaire pour comparer le nombre d'occurrences (figure 2).

\section{Composition du corpus en nb de mots et pondération}

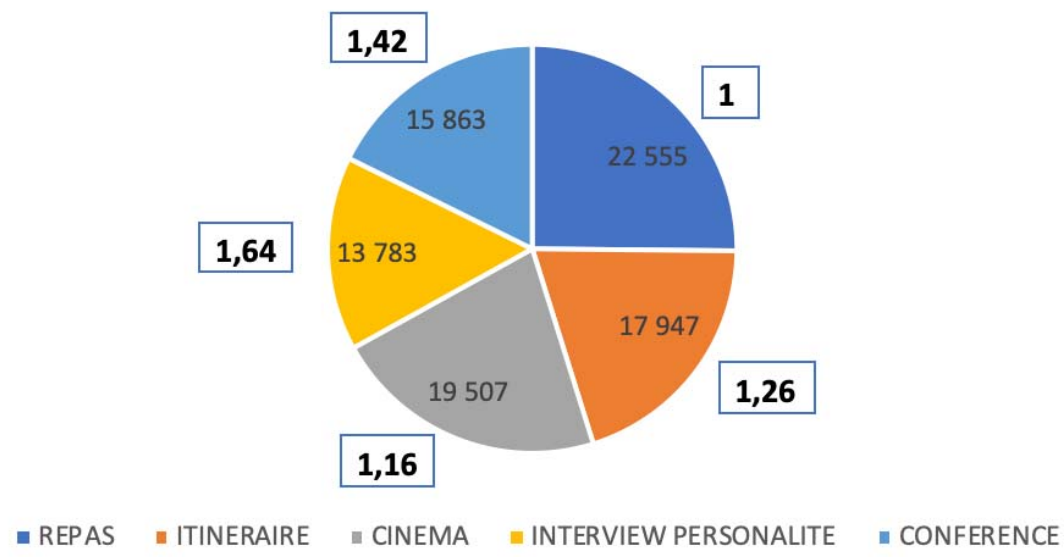

Fig. 2 : Composition du corpus d'étude et pondération de chaque sous-corpus 
L'annotation a été réalisée suite à un repérage manuel des occurrences. Les traits annotés permettent d'interroger la structure des différents types de dislocation ainsi que plusieurs hypothèses sur leurs fonctions pragmatiques. Ainsi, en plus de la position de la dislocation (à gauche ou à droite), ont été annotées la fonction syntaxique (sujet, complément non prépositionnel, complément prépositionnel, locatif) et la catégorie morphosyntaxique ou syntaxique (pronom, SN, Sprep, proposition, etc.) du constituant disloqué. Par ailleurs, le type d'opération sur la structure informationnelle a été examiné pour vérifier les hypothèses sur les fonctions pragmatiques de topicalisation ou de réactivation topicale, et de focalisation. Nous avons également pris en compte les cas d'absence d'opération sur la structure informationnelle. Enfin, malgré l'absence d'analyse prosodique fine, la présence d'une pause entre l'élément disloqué et les constituants centraux a également été relevée.

\section{Caractéristiques des dislocations dans la sélection ESLO : quelques tendances quantitatives}

Les données annotées montrent, comme il est visible dans la figure suivante, que la fonction syntaxique de l'élément disloqué est majoritairement sujet. Le complément direct arrive loin derrière en deuxième position, avant le complément locatif et les compléments obliques spécifiques dont l'objet indirect.

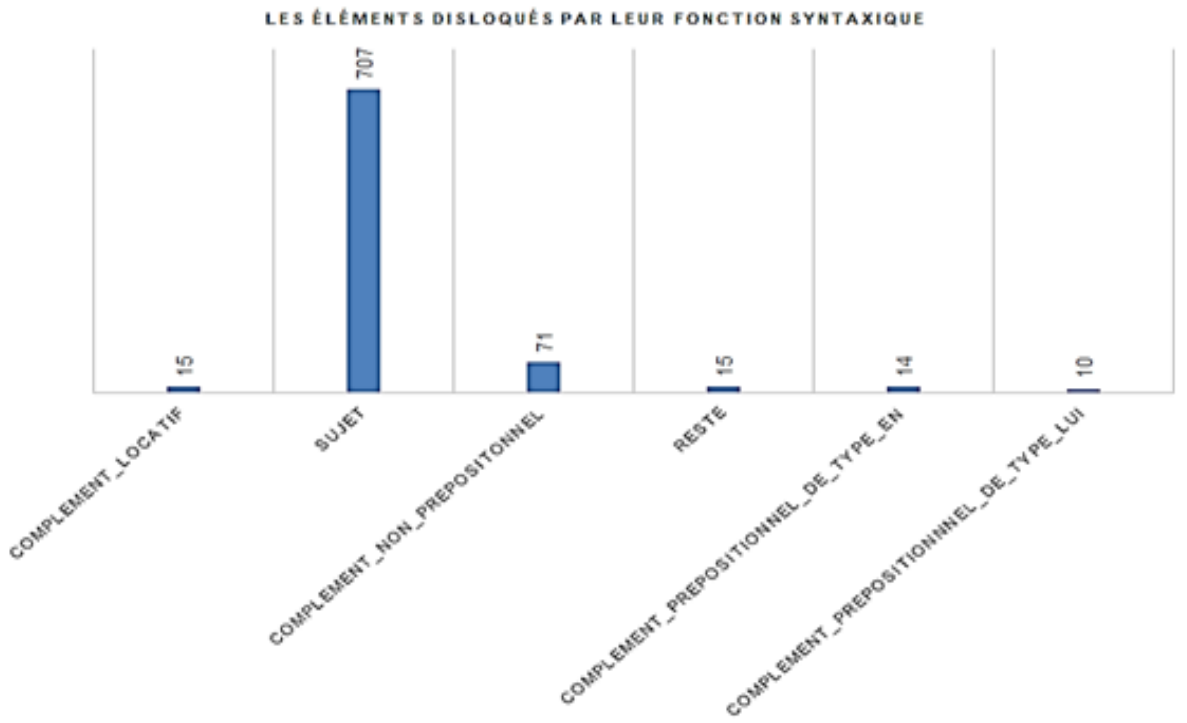

Fig. 3 : Fonctions syntaxiques des éléments disloqués

En ce qui concerne la catégorie morphosyntaxique ou syntaxique du constituant disloqué (cf. schéma ci-dessous pour la dislocation à gauche), la grande majorité relève du domaine nominal : les pronoms, noms propres, SN représentent presque $94 \%$ des cas. Il est intéressant de constater qu'il s'agit en majorité de pronoms $(42 \%)$, mais également de syntagmes nominaux (36,7\%), en particulier de SN définis (35\% contre $1,7 \%$ seulement de $\mathrm{SN}$ indéfinis). Ces résultats peuvent être corrélés à ceux portant sur la structure de l'information. 


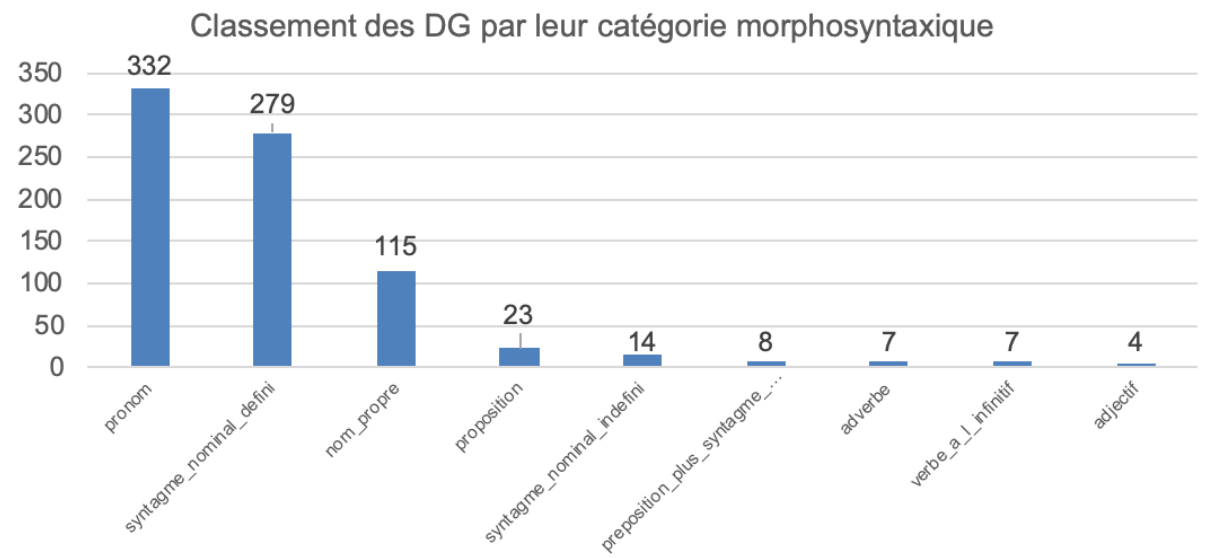

Fig. 4 : Catégories morphosyntaxiques et syntaxiques des éléments disloqués

Les résultats concernant les opérations sur la structure de l'information permettent en effet plusieurs constats. On observe dans un premier temps que la topicalisation est d'une part une fonction commune aux deux types de dislocations et d'autre part la fonction pragmatique principale des dislocations, qu'elles soient à gauche ou à droite $(67 \%$ pour les DD et $64 \%$ pour les DG). Cette tendance peut être mise en perspective avec l'analyse morphosyntaxique : en effet, les catégories morphosyntaxiques et syntaxiques les plus représentées, à savoir les pronoms et les SN définis, sont précisément des techniques permettant d'assurer, entre autres, la coréférence avec un constituant déjà introduit précédemment ou inférable du contexte.

Par ailleurs, on trouve une proportion non négligeable de DG et DD pour lesquels ne semble être à l'œuvre aucune opération sur la structure de l'information, $31 \%$ et $25,2 \%$ respectivement. Cela s'avère particulièrement caractéristique pour les dislocations de type «moi je». Dans ces cas précis, la redondance du sujet sert surtout à des fins interactionnelles de prise de parole, comme il apparaît très clairement dans l'exemple (14). (14)

DS952: ouais mais c'est pas c'est pas pareil moi j'avais trop peur là qu'il y ait quelqu'un qui rentre en plus tu as tout qui est fermé donc tu vois pas dehors moi j'entendais des bruits et tout j'aimais pas

DS952MER: moi j'ai pas peur

DS952SOE: bah moi la dernière fois je suis

DS952MER: ah nan mais moi comme je sais qu'il y a le chien

DS952SOE: bah moi moi j'aime pas ça

\section{ESLO2_REPAS_1256}

Ce résultat peut être interprété comme allant dans le sens de l'hypothèse formulée par certains auteurs (Berrendonner 1993, Zribi-Hertz 1994, cités par Brunetti et al. 2012) qui postulent l'existence d'une particularité morphosyntaxique du français parlé : selon eux, les clitiques ne présenteraient pas les propriétés d'un pronom mais d'un affixe verbal, ce qui remettrait en question l'analyse syntaxique en termes de dislocation. De fait, étant donné que dans un quart à un tiers des occurrences de notre corpus la dislocation n'effectue aucune opération de topicalisation ou de focalisation, on peut s'interroger sur la stabilité de la fonction pragmatique habituellement associée à cette construction et envisager cette réduction fonctionnelle, observable dans une partie des cas, sous l'angle de la grammaticalisation pour formuler l'hypothèse d'un changement en cours. Pour autant, il n'est pas possible de conclure à un fonctionnement systématique du clitique comme affixe, compte tenu du nombre toujours dominant de cas pour lesquels la dislocation représente 
une structure marquée, c'est-à-dire permettant une opération pragmatique spécifique de topicalisation ou de focalisation ${ }^{4}$.

Enfin, les résultats montrent aussi que les dislocations ne sont pas associées massivement à des disfluences ou des reformulations (seulement 7,4\% pour les DG et 3,9 $\%$ pour les $\mathrm{DD}^{5}$ ), ce qui semble indiquer d'une part que la construction ne nécessite pas d'effort cognitif accru, et d'autre part qu'elle n'est pas utilisée comme une ressource à l'appui du travail de formulation en temps réel. En revanche, comme nous le verrons plus loin, elle semble être mobilisée à des fins interactionnelles dans la gestion de la clôture de certains échanges et dans les épisodes narratifs, au sein desquels la topicalisation d'objets de discours est particulièrement présente.

\section{DG / DD : un curseur sur le continuum proximité - distance à l'oral?}

Un autre résultat n'ayant pas été mis en évidence, à notre connaissance, dans des études antérieures, a retenu notre attention car il montre l'effet du contexte sur le choix des ressources langagières et illustre à ce titre la variation diaphasique au niveau syntaxique. Nous avons en effet pu comparer, dans les sous-corpus sélectionnés, les fréquences d'emploi respectives des dislocations à droite et des dislocations à gauche, ce qui donne un rapport de fréquence intéressant. On peut d'abord observer dans la figure 5 que la fréquence des DD (en orange) baisse graduellement à mesure que l'on se rapproche de la distance communicative : ainsi, alors que les DD sont au nombre de 82 dans les repas, on n'en compte plus que 4 dans les conférences universitaires. On observe pour les DG (en bleu) également une différence quantitative, même si ces chiffres ne suivent pas une courbe décroissante régulière : les DG sont beaucoup plus nombreuses dans les repas (227 occurrences) que dans les autres contextes (de 63 à 94 occurrences).

De plus, on notera également que les doubles dislocations, certes peu nombreuses (12 dans tout le corpus) connaissent une répartition qui relève de la même tendance. En effet 10 d'entre elles sont relevées dans les repas entre proches, 2 à la sortie du cinéma, mais aucune dans les contextes plus formels.

\footnotetext{
${ }^{4}$ La question qui reste néanmoins posée est celle de la fréquence des différentes opérations de topicalisation évoquées en 1 (réactivation d'un topic, introduction d'un nouveau topic, d'un topic dérivé, contraste) et celle d'une possible distribution entre l'une et l'autre structure. Une annotation plus précise devrait être entreprise pour interroger les hypothèses en cours sur les différents types de topicalisation, notamment la question d'une spécification pragmatique respectivement pour la dislocation à gauche et la dislocation à droite (cf. Pekarek-Dohler 2001 pour une discussion). De plus, il serait intéressant d'interroger cette éventuelle répartition fonctionnelle au regard de la diversité des situations d'échange représentées au sein du sous-corpus.

${ }^{5}$ A titre de comparaison, la réalisation des relatives en que, où, dont et les composés de lequel, est accompagnée de disfluences dans respectivement $11,9 \%, 11,4 \%, 17,6 \%$ et $14,3 \%$ des cas dans l'échantillon analysé par Larrivée \& Skrovec (2019).
} 


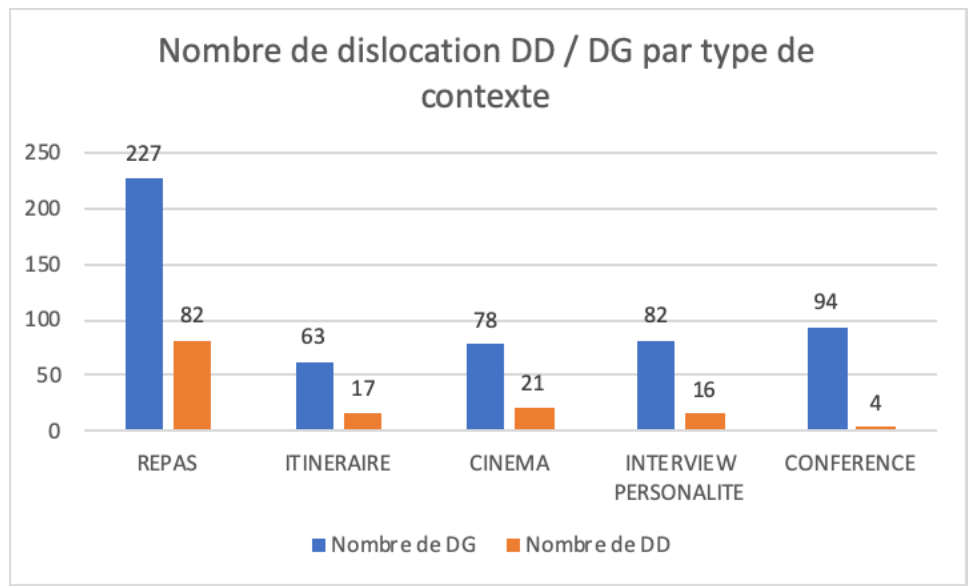

Fig. 5 : Nombre de dislocations à gauche et dislocations à droite par type de contexte

Mais c'est surtout lorsque l'on examine le rapport de fréquence entre DG et DD, c'està-dire leur fréquence relative, que l'on fait le constat le plus frappant, celui d'une corrélation entre cette fréquence relative et le continuum proximité-distance communicative. On observe ainsi (figure 6) que plus le contexte est caractérisé par la familiarité des locuteurs, un fort degré d'interactivité, peu de préparation thématique et un faible degré de formalité, moins l'écart est élevé entre le nombre de DG et le nombre de DD. A l'inverse, plus le cadre est formel, avec des interactants inconnus et un niveau d'interactivité faible, plus l'écart se creuse. Le nombre de DG par rapport aux DD est respectivement 2,8 fois supérieur dans les repas, 3,7 dans les demandes d'itinéraires et les micro-trottoirs à la sortie du cinéma, 5,1 dans les interviews et 23,5 dans les conférences universitaires.

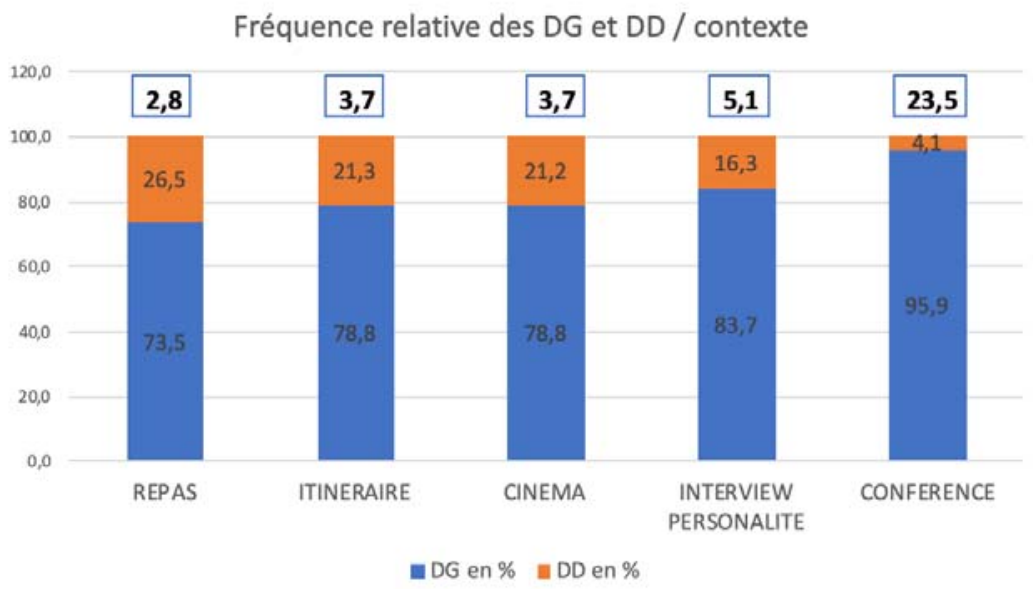

Fig. 6 : Fréquence relative des DG et DD par rapport au contexte : pourcentages de fréquence et indices respectifs

Ainsi, le rapport de fréquence relative des deux types de dislocations dans notre corpus d'étude reflète le continuum variationnel proximité - distance. Ce constat mérite certes d'être confirmé par un examen dans un corpus plus important, mais il est intéressant dans la mesure où il fait écho à d'autres résultats obtenus sur des objets linguistiques différents et mettant également en évidence l'existence d'un continuum variationnel au niveau des formes linguistiques. Larrivée \& Skrovec (2019) montrent ainsi, à propos des relatives, une 
corrélation inverse : leur fréquence baisse à mesure que l'on s'approche de la proximité communicative, c'est-à-dire dans des contextes plus informels et interactifs.

\section{Les dislocations comme stratégies interactionnelles}

Une analyse qualitative des contextes interactionnels larges dans notre corpus a permis de mettre en évidence l'importance des dislocations comme stratégies interactionnelles visant à accomplir les tâches communicatives précises.

D'une part, c'est dans l'organisation séquentielle des interactions, et notamment l'organisation de la phase de clôture, que cette construction semble jouer un rôle. Étant donné qu'une partie du corpus est composée de micros-trottoirs à la sortie du cinéma et de demandes d'itinéraires, il était relativement facile de déterminer le rôle interactionnel des énoncés réalisés sous le format disloqué. Ainsi, nous avons relevé, dans 4 enregistrements différents, 4 occurrences de la dislocation réalisées dans le but d'expliquer la raison d'être du micro-trottoir, dirigeant ainsi la conversation vers sa fin, comme dans l'exemple (15) cidessous. Notre sous-corpus « cinéma » étant composé de 37 enregistrements, la dislocation est donc utilisée dans 10,8\% des enregistrements de ce type. Le double marquage étant un procédé rare (Blasco-Dulbecco 1999 : 111), nous nous permettons de constater qu'il n'y a pas de différence significative quant à la fréquence de son utilisation dans cette situation de communication par rapport à celle en général. Dans l'exemple suivant, la locutrice BL828 utilise le format disloqué comme un moyen de préclôture topicale.

L828: d'accord donc en fait ces enregistrements ils vont nous servir parce qu'on dresse le le portrait en fait sonore de la ville d'Orléans en deux mille onze oui donc on voulait vous demander si c'était possible de d'utiliser cet enregistrement en fait c'est totalement anonyme d'accord?

HS609: y a pas de soucis

VP283: y a pas de soucis

BL828: d'accord bah je vous remercie beaucoup

HS609: et c'est pour quelle fac

BL828: bonne soirée

ESLO2_CINE_1179

Par ailleurs, la dislocation à gauche est repérable dans les séquences narratives de l'oral spontané, au sein desquelles elle joue, par topicalisation, un rôle de balisage référentiel. Ainsi, nous avons relevé des cas où les interactants ont recours au format disloqué à gauche pour enchaîner les actions successives des « acteurs » principaux de l'épisode relaté :

DC040 :ça me fait penser quand j'étais en Angleterre sur le concert des Pigeon Detectives y avait une meuf qui avait été placée sur le côté des barrières qui a voulu chop- monter sur le sur la scène le vigile il l'a vue boum il lui a mis un contre la barrière [rire en fond]

FG719: placage comme euh

PF437: comme la pub avec le footballeur américain là

DC040: mais trop ça

DC040: la meuf elle s'est mangé la barrière après elle s'est relevée elle a voulu se barrer

le vigile il est arrivé boum deuxième tampon elle est tombée par terre il l'a défoncée la meuf [rire] j'ai regardé ma pote je lui fais ben je veux pas monter sur scène hein ESLO2_REPAS_1265

Dans la séquence de l'exemple (16), il est bien visible que la dislocation sert notamment à marquer l'alternance des agents au sein de l'épisode narratif. La première référence au personnage féminin est actualisée par une présentative existentielle ( $y$ avait une meuf) contenant un $\mathrm{SN}$ objet indéfini. La mention du vigile (déjà évoqué en amont) 
dans une dislocation à gauche, le réactualise comme agent des actions qui suivent, introduites par simple pronominalisation. Lorsque l'agent change (la meuf), la référence au personnage féminin est actualisée par une nouvelle dislocation à gauche, suite à quoi le locuteur formule trois actions successives de celle-ci avec sujet pronominal. La dislocation suivante sert à marquer un nouveau changement de topique sujet pour référer à des actions effectuées par l'autre personnage, le vigile.

Néanmoins, bien que nous ayons trouvé des occurrences se situant dans ce cadre et que nous sommes sûrs que ce format est utilisé pour raconter des histoires ou des synopsis des films, il est nécessaire dans les études ultérieures de quantifier leur fréquence et idéalement leur fréquence dans les interactions ayant les mêmes caractéristiques, afin de comprendre s'il s'agit d'une stratégie privilégiée ou accessoire.

\section{Conclusion}

Bien qu'elle ait pour son objet un sujet abondamment traité par les linguistes de différents courants, cette étude essaye d'apporter sa pierre à l'édifice en exploitant un corpus diversifié, comportant des enregistrements faisant varier plusieurs dimensions discursives, le corpus ESLO. Le traitement pluridimensionnel de ces données nous a permis un éventail de possibilités pour leur analyse en mettant l'accent sur les aspects syntaxique, pragmatique et interactionnel.

La définition du phénomène de la dislocation ne fait pas unanimité parmi les chercheurs, même quand il s'agit du double marquage (Berrendonner 2015). Nous nous sommes finalement fondés sur une définition large du phénomène inspirée des travaux de Mylène Blasco-Dulbecco $(1999,2006)$, tout en écartant certaines structures limitrophes. La particularité de l'approche a consisté à travailler à partir d'un corpus échantillonné illustrant différents genres discursifs entre proximité et distance communicative pour contribuer à décrire ce phénomène à partir de données situées. Notre corpus d'étude a été prélevé dans le corpus ESLO2 et ensuite équilibré par pondération différente de ses sousparties afin de permettre la comparaison et ainsi l'étude de la variation diaphasique de la dislocation. Concernant la fonction syntaxique de l'élément disloqué, nos données montrent une prédominance prononcée des sujets. Quant à la catégorie grammaticale, ce sont les pronoms, suivis des syntagmes nominaux qui sont les plus fréquents. Pour ce qui est de l'opération effectuée sur la structure informationnelle, il s'avère que les dislocations sont majoritairement un instrument de topicalisation. Cependant, vu un pourcentage relativement important d'occurrences qui reste neutre de point de vue de la topicalisation, il n'est pas possible de postuler que la dislocation est nécessairement associée à la topicalisation. Ce constat rejoint les hypothèses sur une tendance du français parlé au double marquage sans valeur pragmatique comme spécificité typologique.

L'apport principal de cette étude se situe au niveau de l'analyse diaphasique. En effet, nous avons démontré que, non seulement le nombre de DD diminue, mais aussi que l'écart entre le nombre de DG par rapport au celui de DD se creuse en faveur des DG à mesure que l'on se rapproche de la distance communicative dans notre corpus, ce qui nous permet de postuler l'existence d'une corrélation négative entre le nombre de DD et la distance communicative. Plus exactement, les DG sont 23,5 fois plus nombreuses que le DD dans nos conférences universitaires, tandis que ce rapport est seulement 2,8/1 pour nos conversations autour d'un repas, plus spontanées. Ainsi, ce constat vient conforter l'hypothèse d'un effet du genre interactionnel sur les choix syntaxiques reflétant le continuum proximité-distance, la corrélation pouvant être positive ou négative en fonction du phénomène syntaxique considéré : chez Larrivée \& Skrovec (2019) plus le contexte est formel et monologué, plus les constructions relatives sont fréquentes; à l'inverse, dans le 
présent échantillon, les dislocations sont plus fréquentes dans les contextes informels et interactifs.

Enfin, notre travail offre des pistes pour l'analyse de l'emploi du format disloqué dans la réalisation des tâches interactionnelles identifiables. Son usage dans le cadre de la préclôture topicale ou celui de la narration est confirmé mais doit être quantifié plus précisément dans les travaux ultérieurs afin d'en déduire son poids relatif.

\section{Références}

Abouda, L. \& Baude, O. (2007). Constituer et exploiter un grand corpus oral : choix et enjeux théoriques. Le cas des Eslo. In F. Rastier et M. Ballabriga (dir.), Corpus en Lettres et Sciences sociales. Des documents numériques à l'interprétation, Actes du XXVIIe Colloque d'Albi : 161168.

Avanzi, M. (2009). La dislocation à gauche avec reprise anaphorique en français parlé : Étude prosodique. Interface Discours \& Prosodie Paris, 9-11 septembre 2009.

Bally, C. (1909). Traité de stylistique française. 2e édition, Heidelberg : Carl Winter's Universitätsbuchhandlung.

Baude, O. \& Guerin, E. (2015). Pourquoi et comment dresser le portrait sonore d'une grande ville ? L'exemple d'ESLO 2. In Gadet, F. (éd.), Actes du colloque Les métropoles francophones en temps de globalisation.

Berrendonner, A. \& Béguelin, M.-J. (1997). Left-dislocations, varieties, use and norm, in Cheshire. J. \& Stein, D. (éds.) Taming the vernacular, London : Longmann.

Berrendonner, A. (1993). Sujets zéro. In : Complétude et incomplétude dans les langues romanes et slaves. Actes du VI colloque international, Cracovie, Sept. 1991, WSP, Cracovie, 17-45.

Berrendonner, A. (2008). Dislocation et conjugaison. Cahiers de Praxématique 48, 85-110.

Berrendonner, A. (2015). Constructions disloquées. In Encyclopédie grammaticale du français, en ligne : encyclogram.fr

Blanche-Benveniste, C. (1996). Trois remarques sur l'ordre des mots dans la langue parlée. Langue française, $n^{\circ} 111$, 1996. L'ordre des mots, sous la direction de Henning Nølke et Hanne Korzen, 109-117.

Blasco-dulbecco, M. (1999). Les dislocations en français contemporain, étude syntaxique. Paris : Honoré Champion.

Blasco-Dulbecco, M. (2006). Propositions pour le classement typologique de quelques détachements. In : L'Information Grammaticale, N. 109, 2006. 27-33.

Brunetti, L., Avanzi, M. \& Gendrot, C. (2012). Entre syntaxe, prosodie et discours : les topiques sujets en français parlé. SHS Web of Conferences 1 2041-2054 (2012). DOI: $10.1051 /$ shsconf $/ 20120100209$

Cinque, G. (1977). The Movement Nature of Left Dislocation. Linguistic Inquiry 8.2.

Chafe, W. (1976). Givenness, contrastiveness, definiteness, subjects, topics, and point of view. In: Charles N. Li (ed.) Subject and Topic, New York: Academic Press, 25-55.

Grosu, A. (1975). On the status of positionally-defined constraints in syntax. Theoretical Linguistics, Volume 2, Issue 1-3, 159-201.

Deulofeu J. (1977). La syntaxe et les constructions binaires. Recherches sur le français parlé, 1, 3061.

De cat, C. (2007). French dislocation. Interpretation, syntax, acquisition. UP : Oxford.

Dugua, C. \& al. (2019). Des liaisons et des corpus : apport d'une étude sur le changement linguistique en temps réel. Présentation aux Journées de Linguistique de Corpus, Grenoble, 26-28 novembre 2019.

Grobet, A. (2002). L'identification des topiques dans les dialogues. : Louvain-la-Neuve : De Boeck Supérieur.

Havers, W. (1925). Der sog. 'Nominantivus pendens'. Indogermaniche Forschungen, 43 (2), $207-$ 257.

Horlacher, A. (2015). La dislocation à droite revisitée : Une approche interactionniste. Louvain-laNeuve: De Boeck Supérieur. 
Koch, P. \& Oesterreicher, W. (2001). Gesprochene Sprache und geschriebene Sprache / Langage parlé et langage écrit. In: G. Holtus, M. Metzeltin, Ch. Schmitt (éds), Lexikon der Romanistischen Linguistik, Bd. I/2, 584-627.

Lagorgette, D. (1998). Désignatifs et termes d'adresse dans quelques textes en moyen français. Thèse de doctorat non publiée. Université de Paris X : Nanterre.

Lambrecht, K. (1996a). Information structure and sentence form: Topic, focus, and the mental representations of discourse referents. Cambridge, University Press.

Lambrecht, K. (1996b), Compositional vs. Constructional Meaning: The Case of French comme-N. In T. Galloway \& M. Simons (éds.), Proceedings of the 5th SALT Conference. Ithaca, NY : Cornell University, 186-203.

Lambrecht, K. (2003). On the interaction of formal structure and information structure in grammar. The French detached comme- $N$ construction. In Jam-Ola Östman \& Mirjam Fried (eds), Construction grammar(s). Cognitive and cross- language dimensions, Amsterdam, Benjamins, 2003a, pp. 157-199.

Larrivée, P. \& Skrovec, M. (2019). Variation en français vernaculaire : le cas des relatives. L'Information Grammaticale $n^{\circ} 163,21-28$.

Lehmann, S. (2010). L'évolution des termes d'adresse à contenu social en ancien et en moyen français. Corela [En ligne], HS-8 | 2010, mis en ligne le 11 novembre 2010, consulté le 05 janvier 2020. URL : http://journals.openedition.org/corela/1610 ; DOI : 10.4000/corela.1610

Marchello-nizia, C. (1998). Dislocations en ancien français : thématisation ou rhématisation ? Cahiers de praxématique 30, Montpellier : Pulm. 161-178

Milner, J.C, (1989). Introduction à une science du langage, coll. Travaux linguistiques, Le Seuil : Paris.

Nølke, H. (1998). Il est beau le lavabo, il est laid le bidet : pourquoi disloquer le sujet ? In M. Forsgren, K. Jonasson \& H. Kronning (éds.), Prédication, assertion, information. Uppsala : Acta Universitatis Upsaliensis, 385-393.

Pekarek-doehler, S. (2001). Dislocation à gauche et organisation interactionnelle. Marges Linguistiques, ${ }^{\circ} 2$, 177-194.

Prévost, S. (2003). Détachement et topicalisation : des niveaux d'analyse différents. Cahiers de praxématique 4, Montpellier : Pulm. 97-126.

Rossi, M. (1999). L'intonation, Le système du français : description et modélisation. Paris : Ophrys.

Söll, L. (1974). Gesprochenes und geschriebenes Französisch. Berlin: Erich Schmidt Verlag.

Zribi-hertz, A. (1994). The Syntax of nominative clitics in Standard and Advanced French. In Cinque, G., Koster, J. Pollock, J.-Y., Rizzi, L. \& Zanuttini, R. (éds). Paths Towards Universal Grammar, Georgetown : University Press, 453-472. 